\title{
Electromagnetic Wave Penetration of Reentry Plasma Sheaths
}

\author{
M. P. Bachynski \\ Contribution From the RCA Victor Research Laboratories, Montreal, Canada
}

(Received August 17, 1964; revised September 11, 1964)

\begin{abstract}
Methods to enable electromagnetic waves to propagate through the sheath of plasma created by a high speed vehicle reentering a planetary atmosphere are reviewed and compared. Attention is paid to the possibilities of employing very high frequency waves, low frequency waves, of creating magneto-ionic wave modes by imposing static magnetic fields, and of modifying the plasma by chemical seeding and aerodynamic effects. None of the techniques are without major limitations and considerable experimental studies are still required to establish their feasibility.
\end{abstract}

\section{Introduction}

A space vehicle moving at hypersonic velocities within a planetary atmosphere will be surrounded by a shock-induced layer of ionized gas. This enveloping ionization, or plasma sheath, can have a profound influence on communications and telemetry to and from the vehicle resulting in very high signal attenuation, or "blackout" of the radio signal.

The magnitude of this effect depends upon the flight trajectory in altitude and velocity and upon the shape of the vehicle. Typical altitude-flight velocity variations to be expected for a number of missions are shown in figure 1. Over this range of flight parameters the electron density in the stagnation region [Bachynski et al., 1960] can vary from a few electrons $/ \mathrm{cm}^{3}$ to $10^{18}$ electrons $/ \mathrm{cm}^{3}$ while the electron collision frequency can be as high as $10^{13} \mathrm{sec}^{-1}$. The ratio of plasma frequency to collision frequency can range from very large to very small compared to unity. The thickness of the plasma sheath again depends on vehicle shape, velocity and altitude but as an order of magnitude estimate for a blunt body is 5 to 10 percent of the body radius at the stagnation point and somewhat larger at positions off the stagnation region. In the stagnation region, under conditions of thermal equilibrium, the electron density distribution may be relatively uniform but outside the stagnation region the electron density enveloping the body is very nonuniform and can vary several orders of magnitude along a normal from the body to the bow shock created by the vehicle. The corresponding variation in collision frequency is quite small and for most purposes can be considered uniform along a given normal. Both electron density and collision frequency vary at different distances along the body.

It is these extreme values of the electron density and collision frequency in the plasma sheath and their variation during a flight which affect the interaction of an electromagnetic wave with the plasma and hence seriously interfere with the normal performance of data telemetry, monitoring and command, and control systems operating between the space craft and the ground or between vehicles in flight. Elimination, or at least minimization of these effects is essential, particularly during predetermined positions along the flight path in order to maintain communications with the reentering vehicle.

A number of techniques have been suggested in order to alleviate the "blackout" situation and permit a radio signal to penetrate through the reentry sheath of plasma. In this paper, the possibilities and limitations of using very high frequency radio waves, low frequency radio waves, of imposing static magnetic fields to create magnetoionic mode "windows," and of modifying the plasma by chemical seeding or aerodynamic shaping of the the vehicle are assessed. No single technique appears to offer universal advantages over the others and considerable experimental data is yet required in order to establish the feasibility of a given technique under flight conditions.

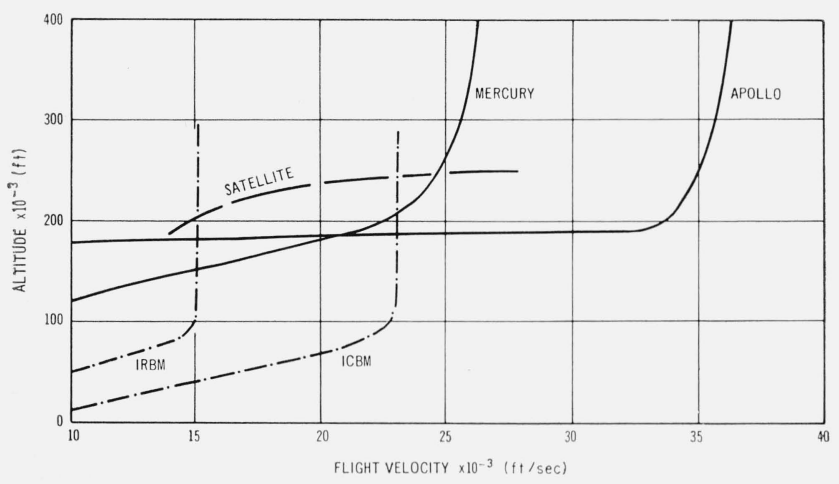

FIGURE 1. Altitude-velocity flight regimes for typical reeniry trajectories. 


\section{Penetration of Reentry Plasma Sheaths by Electromagnetic Waves}

\subsection{Very High Frequencies for Penetrating Plasma Sheath}

Since the refractive index $(\mu)$ of an isotropic uniform lossless plasma is given by

$$
\mu=\left(1-\omega_{p}^{2} / \omega^{2}\right)^{1 / 2}
$$

where: $\omega_{p}=\sqrt{n e^{2} / m \epsilon_{0}}$ is the plasma frequency, $\omega$ is the radian radio frequency, $n$ is the electron number density, $e, m$ are the electronic charge and mass respectively,

$\epsilon_{0}$ is the permittivity of free space,

it is immediately evident that if $e-m$ wave propagation through a layer of plasma is desired, then it can be achieved by the choice of an appropriately high radio frequency such that the refractive index of the plasma will not deviate appreciably from unity. Such a radio frequency requires $\omega>\omega_{p}$ or $f>8,970 \sqrt{n}$. The peak electron densities anticipated would thus determine the choice of frequency. The influence of collisions between the electrons and other species tends to absorb some of the $e-m$ energy and boundaries create losses due to reflection of some of the incident field, but nevertheless a fraction of the incident energy would penetrate through the plasma.

In figure 2 is shown the variation of frequency with electron density corresponding to the "cutoff" or $\omega=\omega_{p}$ condition. The most noteworthy feature

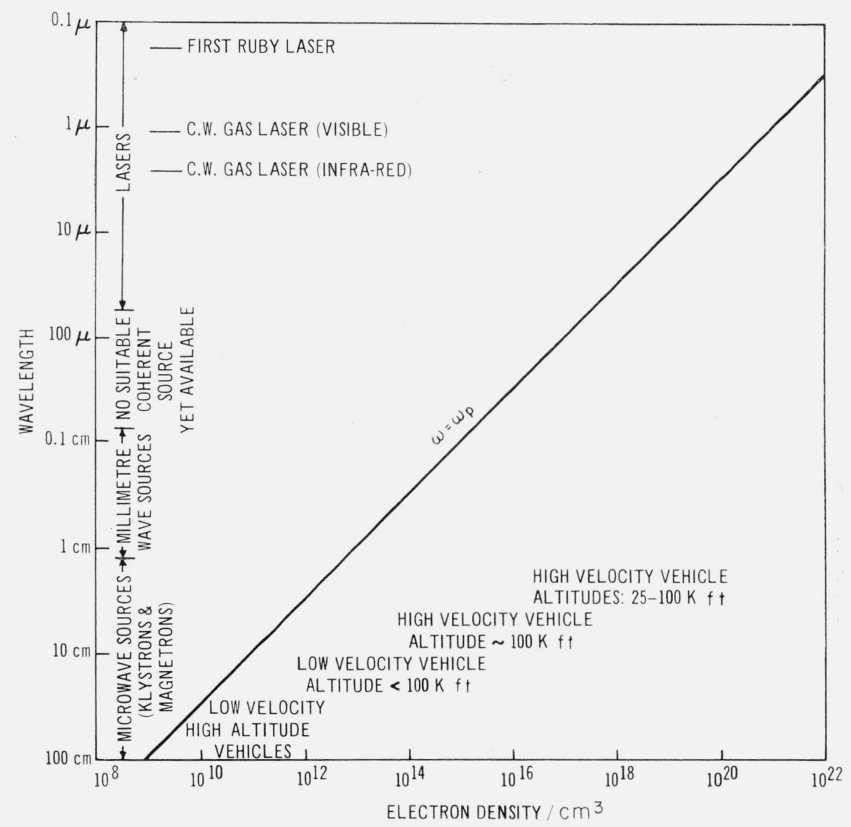

Figure 2. Variation of electron density with radio frequency such that $\omega=\omega_{\mathrm{p}}$ showing the wavelength corresponding to the plasma densities created by hypersonic vehicles at various altitudes and velocities. is the high frequencies corresponding to millimeter and submillimeter wavelengths, required in order to insure communication during reentry. At these high frequencies absorption of electromagnetic waves in the surrounding planetary atmosphere due to free molecules and suspended particles must be considered. In the earth's atmosphere, for instance, absorption of $e-m$ energy is due to the interaction of the incident rf fields with the magnetic dipole of oxygen and the electric dipole of water vapor. As a result abnormally high attenuation occurs at certain frequencies due to these resonance effects. In particular, the absorption due to oxygen is very large for waves of 0.5 to $0.25 \mathrm{~cm}$ wavelength and due to water vapor for wavelengths of the order of $1.3 \mathrm{~cm}$ to less than $0.2 \mathrm{~cm}$. The absorption due to water vapor is directly proportional to vapor content. At wavelengths below $1 \mathrm{~mm}$, the absorption by water vapor in the atmosphere is so high as to make such wavelengths of little value for free space transmission except over very short distances. Typical variations with frequency of the absorption at sea level [after Warren et al., 1962] are shown in figure 3.

A more useful consideration for communications between the vehicle and an earth based station is the total attenuation suffered by millimeter wave frequencies in passing vertically through the atmosphere [Warren et al., 1962] shown in figure 4. As can be seen, the total attenuation is not as great as might at first be expected since the effective thickness of the atmosphere in terms of air at sea level is only a few kilometers.

Several other considerations in regard to millimeter and submillimeter frequencies should be kept in mind. At frequencies which are relatively high compared to the rotational frequencies of the water molecule, little absorption occurs and the medium is again "transparent." Thus optical laser techniques

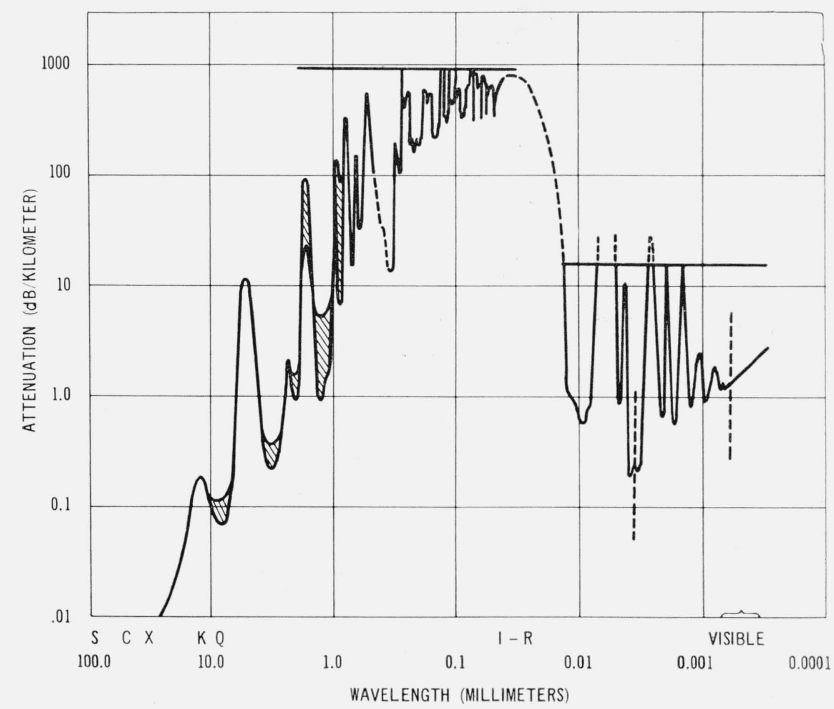

FiguRE 3. Attenuation for one way transmission through air at sea level for region from radio to visible frequencies.

(After Warren et al. [1962].) 


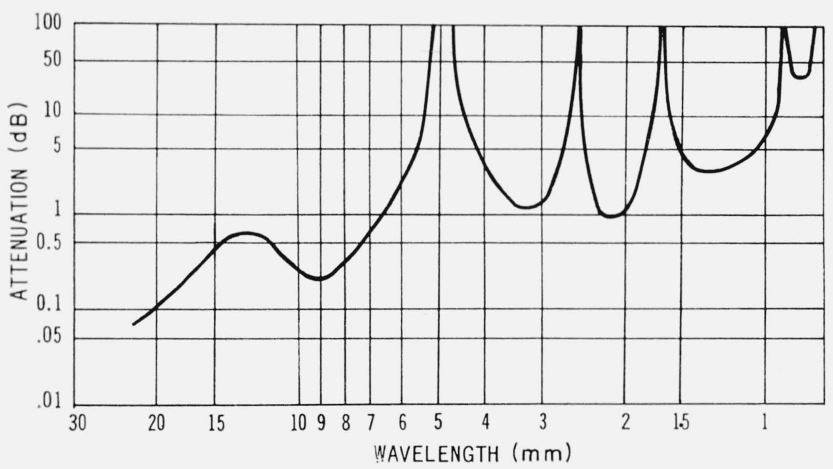

FiguRe 4. Approximate attenuation for vertical radio transmission (one way) through the earth's atmosphere as function of frequency.

for reentry communications should be seriously examined. The absorption due to oxygen is strongly dependent upon pressure (altitude). Hence although air-to-ground paths may present prohibitively high attenuation due to oxygen absorption, air-to-air communications may indeed be practical. (The water vapor content of the atmosphere decreases rapidly with altitude so that at high altitudes absorption due to water vapor is negligible.) A further consideration is the power output of the sources and the weight and size of the associated power supply [see, for example, Shkarofsky and Brannen, 1962]. For millimeter and submillimeter frequencies, the general rule is low power output and bulky high voltage power packs.

In any event, the absorption in the atmosphere due to water vapor, oxygen, and precipitation (liquid or solid form), should they be present, plays an important role in the selection of an optimum high frequency for reentry communications. For reentry into planetary atmospheres, other than that of the earth, similar considerations [Evans et al., 1962] must be paid to the $e-m$ wave absorption of the corresponding atmosphere.

\subsection{Penetration of the Plasma Sheath at Low Frequencies}

For radio frequencies very near yet below the plasma frequency the depth of penetration $\left(d_{s}\right)$ or skin depth of an electromagnetic wave is given by:

$$
d_{s}=c / \omega_{p}=5.32 \times 10^{2} n^{-1 / 2}
$$

where: $c$ is the velocity of light.

The electron density alone determines the depth of penetration of the wave into the plasma. At frequencies lower than both the plasma frequency and the collision frequency, the plasma behaves as a conducting medium and the skin depth is given by:

$$
d_{s}=c / \omega_{p} \sqrt{2 \nu / \omega}=7.52 \times 10^{2} \sqrt{\nu / \omega n}
$$

where: $\nu$ is the electron collision frequency for momentum transfer. Thus for such a plasma, as the radio frequency is decreased or the collision frequency increased, the skin depth actually increases. Hence in this region of plasma parameters a lower frequency electromagnetic wave is attenuated less than a higher frequency (although still lower than the plasma frequency) wave.

This situation is shown schematically in figure 5 . In the low frequency or conducting region an increase of collision frequency increases the skin depth while an increase in electron density decreases the skin depth. The variation of skin depth normalized to wavelength (i.e., $k d_{s}$ where $k=2 \pi / \lambda, \lambda$ is the free space wavelength) with normalized radio frequency is shown in figure 6 . Because of the normalization the skin depth parameter is a monotonically increasing function and allows ready extrapolation between the values shown.

Hence in the real situation if the plasma enveloping a given portion of a space vehicle is relatively thin (say a few tens of centimeters at most), then this may correspond to a fraction of a wavelength for a low frequency wave. The attenuation of the wave upon passing through the plasma $-e^{-d_{p} / d_{s}}$ where $d_{p}$ is the plasma thickness, may be tolerable. Under these conditions the radio signal will suffer a predetermined attenuation, but since it only traverses a small distance (in terms of skin depth) a useful signal emerges through the plasma. The low frequency idea may be applicable for trajectories where the collision frequency increases as rapidly as the electron density and hence the skin depth does not increase but may even decrease (if $\nu$ varies more rapidly than $n$ ). The other considerations are then the bandwidth of the information which

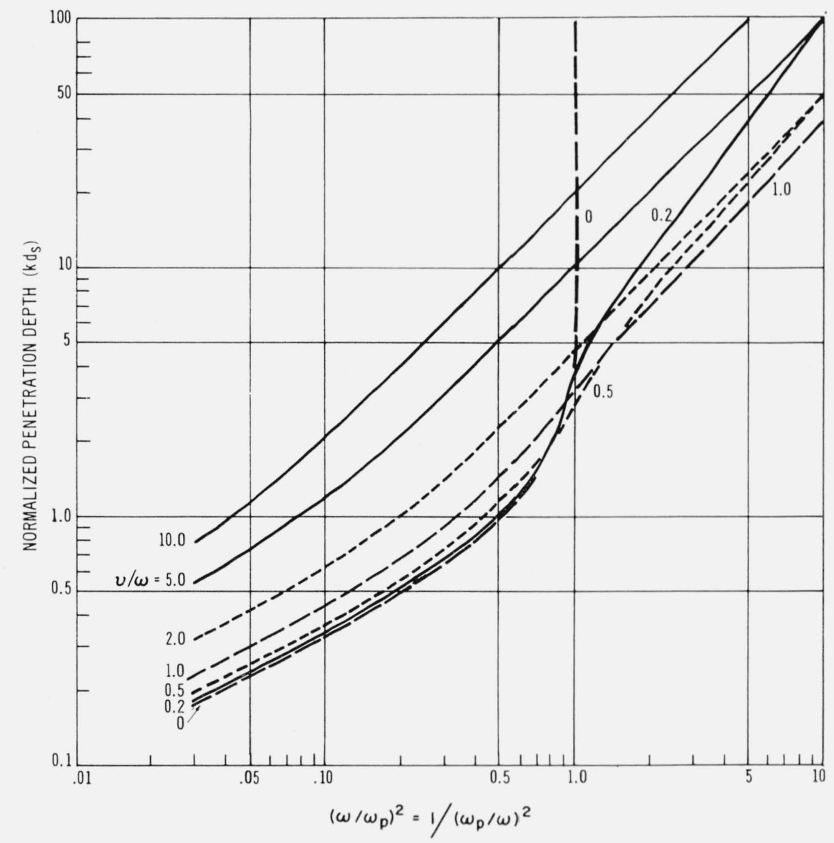

FiguRE 5. Variation of the skin depth or penetration depth of a plasma in the conducting and cutoff regions with electron density and collision frequency. 


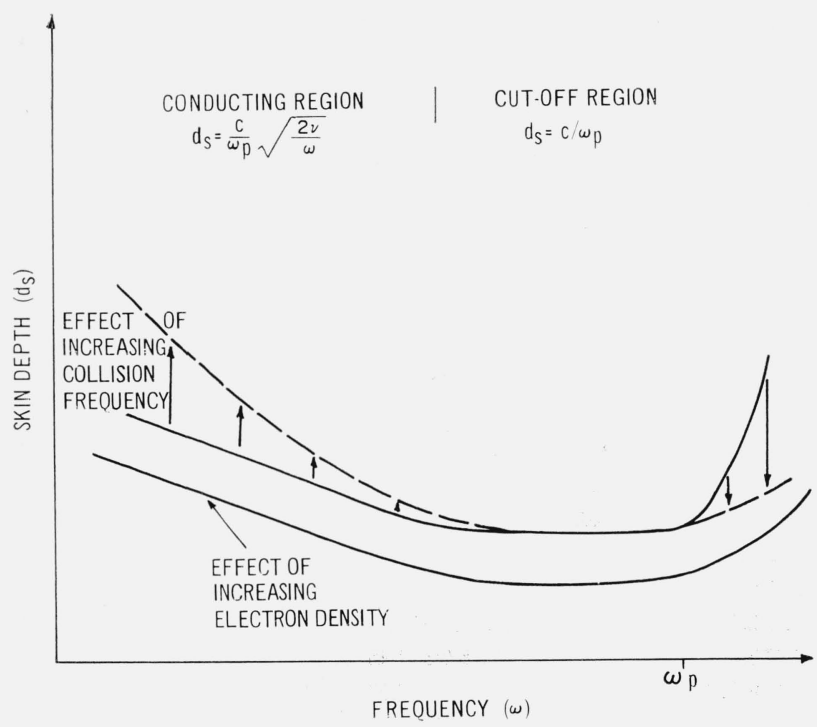

Figure 6. Variation of normalized penetration or skin depth $\left(\mathrm{kd}_{\mathrm{s}}\right)$ of an electromagnetic wave with frequency for different values of collision frequency.

the low frequency signal must carry since a lower frequency signal of necessity implies a narrower bandwidth of possible information, the size of low frequency antennas of any appreciable gain; interference due to atmospheric noise, and the strength of the incident signal (if the transmitter is located on the reentry craft) so as not to appreciably increase the ionization in the plasma sheath. The influence of the ionosphere on low frequency $e-m$ waves must also be kept in mind.

\subsection{Magneto-Ionic Wave Modes for Transmission Through Plasma Sheath}

In the presence of a static magnetic field the variation of the refractive index of the plasma with frequency is governed by the Appleton-Hartree equation which can be written (see for example Allis et al. [1963], Stix [1962], Budden [1961]) :

$$
\begin{aligned}
\mu^{4}\left[\epsilon_{11} \sin ^{2} \theta+\epsilon_{33}\right. & \left.\cos ^{2} \theta\right]-\mu^{2}\left[\left(\epsilon_{11}^{2}-\epsilon_{12}^{2}\right) \sin ^{2} \theta\right. \\
& \left.+\epsilon_{11} \epsilon_{33}\left(1+\cos ^{2} \theta\right)\right]+\epsilon_{33}\left(\epsilon_{11}^{2}-\epsilon_{12}^{2}\right)=0
\end{aligned}
$$

or

$$
A \mu^{4}-B \mu^{2}+C=0
$$

where the coordinate axis has been so chosen that the magnetic field is in the z-direction and the dielectric coefficient of the plasma $(K)$ is given by:

$$
K=\left|\begin{array}{ccc}
\epsilon_{11} & j \epsilon_{12} & 0 \\
-j \epsilon_{12} & \epsilon_{11} & 0 \\
0 & 0 & \epsilon_{33}
\end{array}\right|
$$

and for a lossless plasma

$$
\begin{aligned}
& \epsilon_{11}=1-\frac{\omega_{p}^{2}}{\omega^{2}-\omega_{b}^{2}} \\
& \epsilon_{12}=\frac{\omega_{p}^{2} \omega_{b}}{\omega\left(\omega^{2}-\omega_{b}^{2}\right)} \\
& \epsilon_{33}=1-\frac{\omega_{p}^{2}}{\omega^{2}} \\
& \omega_{b}=\frac{e}{m} B_{0}
\end{aligned}
$$

where: $B_{0}$ is the imposed static magnetic field, $\theta$ is the angle between the direction of propagation and the magnetic field.

For a lossless plasma, conditions exist whereby the refractive index of the plasma is infinite (i.e., there is a "pole" in the expression for the refractive index). Under these conditions the phase velocity of an $e-m$ wave in the plasma is zero. This "resonance" condition corresponds to a very high (infinite in the limiting case) value of the conduction current in the plasma and is associated with strong absorption of $e-m$ energy in the plasma.

Conditions for a lossless plasma also exist whereby the refractive index of the plasma can be zero (i.e., there is a "zero" in the expression for the refractive index). The phase velocity of a wave in the plasma is then infinite. At such "cutoff" conditions the conduction current is just canceled by the displacement current. This condition corresponds to a strong reflection of the electromagnetic fields from such a medium.

Thus when the plasma parameters and the frequency are varied, the refractive index (and hence the wave phase velocity) will vary through these values. (In the case where collisions are present, the resonances and cutoffs are not as sharp as for the lossless case. The refractive index (and phase velocity) in the presence of collisions may, however, pass near the zero and infinite values as the plasma parameters and frequency are varied. When the collision frequency is high $\left(\nu \sim \omega_{p}\right)$ most of this behavior is obscured by high attenuation.)

From (5) poles occur in the dielectric coefficient, provided

$$
A=\epsilon_{11} \sin ^{2} \theta+\epsilon_{33} \cos ^{2} \theta=0 \quad \text { when } C \neq 0 .
$$

The equation for the resonance conditions is then:

$$
\tan ^{2} \theta+\frac{\epsilon_{33}}{\epsilon_{11}}=0
$$

For propagation along the direction of the static magnetic field $[\theta=0]$ this condition occurs at:

$$
\epsilon_{11}=\infty \quad \text { i.e., } \omega^{2}=\omega_{b}^{2} \text {. }
$$

(The $\epsilon_{33}=0$ root has been excluded since this makes 
$C=0$.) For propagation transverse to the magnetic field $[\theta=\pi / 2]$ resonance occurs for $\tan \theta=\infty$ or:

$$
\epsilon_{11}=0 ; \quad \text { i.e., } 1=\omega_{p}^{2} /\left(\omega^{2}-\omega_{b}^{2}\right) \text {. }
$$

Zeros will occur in the dielectric coefficient when $C=0$ (if $A \neq 0$ simultaneously). This leads to the condition for cutoff, namely:

$$
\epsilon_{33}\left(\epsilon_{11}^{2}-\epsilon_{12}^{2}\right)=0
$$

which is independent of angle. Equation (7) thus gives:

$$
\begin{aligned}
& 1=\omega_{p}^{2} / \omega^{2} \\
& 1=\frac{\omega_{p}^{2}}{\omega^{2}\left(1 \pm \frac{\omega_{b}}{\omega}\right)} .
\end{aligned}
$$

The poles and zeros of the dielectric coefficient separate the values of the plasma properties where the dielectric coefficient is negative or positive. In regions between adjacent poles and zeros (resonances and cutoff) where the dielectric coefficient is negative, electromagnetic waves cannot penetrate without severe attenuation. These regions thus designate "stop-bands" for the propagation of $e-m$ waves in a plasma.

A schematic diagram showing the variation of the opaque and transparent regions of an anisotropic plasma with plasma parameters for both the ordinary and extraordinary wave are shown in figure 7 . Figure $7 \mathrm{a}$ is for propagation along the direction of the static magnetic field and figure $7 \mathrm{~b}$ for propagation normal to the direction of the static magnetic field. These "pass" regions depend upon the radio frequency, polarization and angle of incidence of the electromagnetic wave, and upon the electron density and applied static magnetic field. Hence an electromagnetic wave of any frequency can be made to penetrate through the plasma provided a sufficiently strong magnetic field is applied to the plasma.

The above considerations have led to the suggestion [Hodara et al., 1960; Flesher, 1960] of using magnetic fields to alter the plasma characteristics in order to achieve communications using an appropriate magneto-ionic mode.

The value of magnetic field required to open these "windows" in the plasma is defined through the magnitude of the electron cyclotron frequency; namely:

$$
\omega_{b}=\left|\frac{e}{m} B_{0}\right|=17.6 \times 10^{6} B_{0} \text { (gauss) }
$$

The actual magnitude of field necessary will depend upon the wave mode used, the radio frequency of operation and the electron density of the plasma and can readily be calculated. Hodara [1961] has published a number of numerical results for the right-hand circularly polarized wave for propagation in the direction of the applied magnetic field. In general, values of several hundred gauss and greater are required. Hence, weight, size, and power supply considerations become of prime importance. In this regard the use of superconducting materials looks very promising despite the problem of cooling these alloys to very low temperatures.

Mullin [1962] has raised the problem of the energy lost due to the mismatch presented by a finite slab of plasma even when the plasma is transparent once the $e-m$ signal penetrates into the plasma. The effect of the mismatch is to reduce the apparent advantage of the extraordinary wave mode over the ordinary wave mode for propagation along the magnetic field and to cause the transmission properties of the extraordinary wave mode to exhibit sharp peaks (when the optical thickness of the plasma is $m(\lambda / 4), m$ being an integer) with changing plasma density. In the reentry case, the plasma boundaries will not be infinitely sharp so that this effect may not be so severe. Nevertheless it may still be appreciable. Schemes to vary the strength of the magnetic field in order to minimize the mismatch and to shape the magnetic field to compensate for the plasma nonuniformity have also been suggested [Fischer, 1963].
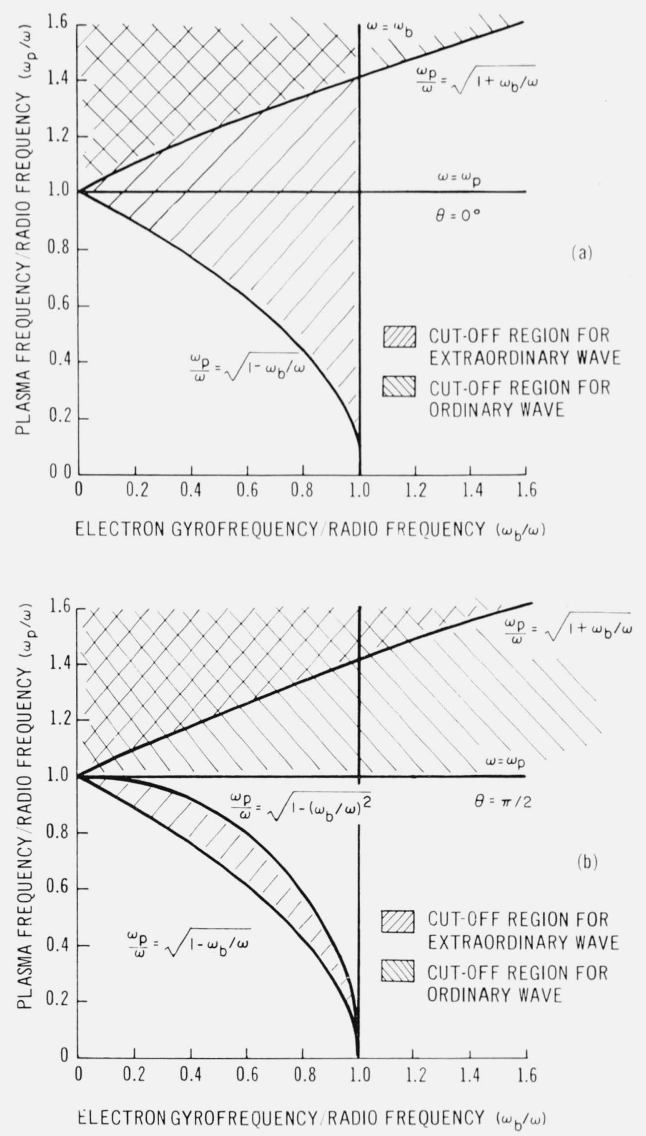

FiguRe 7. Dependence of cutoff regions of magneto-ionic wave modes on plasma frequency and electron gyrofrequency for (a) propagation along the direction of magnetic field $\left(\theta=0^{\circ}\right)$, (b) propagation across the direction of magnetic field $(\theta=\pi / 2)$. 

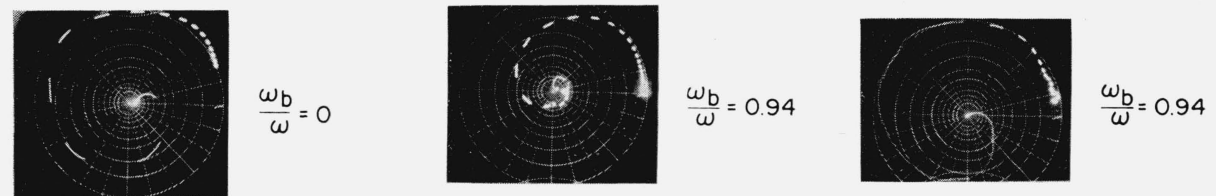

ISOTROPIC PLASMA

Attenuation $=2 \mathrm{db} /$ division of radius vector Phase change $=$ polar angle

Timing marker: $10 \mathrm{kc} / \mathrm{s}$ repetitive rate

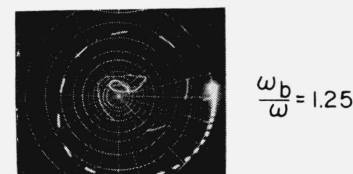

EXTRAORDINARY WAVE (right-hand cicularly polarized)

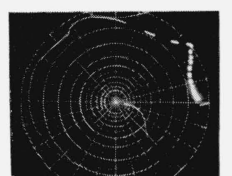

ORDINARY WAVE

(left-hand circularly polarized,

$\frac{\omega_{\mathrm{b}}}{\omega}=1.25$

FIGURE 8. Experimental measurements of transmission of a circularly polarized plane electromagnetic wave through a slab of plasma (1.56 wavelengths thick) of varying electron density generated in helium at a pressure of 0.5 torr.

Plasma density is the same for corresponding timing markers (starting with the innermost).

For propagation along the direction of magnetic field, it is necessary to use circularly polarized $e-m$ waves if Faraday rotation effects are to be avoided. Furthermore, a gain of $3 \mathrm{~dB}$ over a linearly polarized $e-m$ wave may be achieved by using the appropriate circular polarization if the second mode is cutoff under the operating conditions.

Although substantial theoretical work has been done on various aspects of magneto-ionic theory in order to achieve communication during reentry, there exists surprisingly few published measurements on propagation of $e-m$ waves in dense magnetized plasmas [Mahaffey, 1963; Dellis and Weaver, 1964]. Hence experimental techniques for using the magnetic field to alter the plasma, the influence of practical geometric configurations and the degree of accord to be expected between the theory and free-flight experiment is still largely open for investigation. Some indication of the influence of a magnetic field on propagation through a plasma slab has been obtained by Bachynski and Gibbs [1964]. Figure 8 gives a typical example of results for propagation of a circularly polarized plane wave in a plasma along the direction of the magnetic field for a pulsed plasma (varying electron density). For the extraordinary wave at magnetic field strengths such that $\omega_{b} / \omega<1$, the plasma is more opaque than in the absence of a magnetic field. For $\omega_{b} / \omega>1$ the plasma is more transparent than in the isotropic case. (As well, the direction of phase change reverses since $\mu^{2}>1$.) With increasing magnetic field the plasma becomes increasingly transparent to the ordinary wave in the range of densities generated.

(Another method to alter the propagation modes in a plasma either with or without the use of magnetic fields is to shoot an electron beam into the plasma and propagate $e-m$ waves along the direction of the beam. 'This changes the refractive index of the plasma from its isotropic value. No serious considerations have as yet been paid to this technique, primarily due to the problem of coupling the $e-m$ energy into such a system and its technical difficulties.)

\subsection{Modification of Plasma Sheath by Chemical Seeding}

The electromagnetic properties of a plasma can be modified by chemical seeding with additives which

(a) increase the electron density,

(b) decrease the electron density either by

(i) electron attachment to neutral molecules

(ii) decreasing the electron recombination time

(c) change the collision cross section of the species constituting the plasma.

The addition of chemicals which have a very low ionization potential, such as the alkalis, to a plasma in the medium such as air would be expected to enhance the electron density. Calculations by Hochstim [1961] show that the effect of cesium on air is to increase the electron concentration very substantially at low temperatures $(3,000$ to 6,000 。 $\mathrm{K})$. For instance, the addition of one percent cesium to air at temperature $5,000^{\circ} \mathrm{K}$ and density $\rho / \rho_{0}=10^{-3}$ increases the electron concentration by a factor of 50. At temperatures above $8,500^{\circ} \mathrm{K}$ the ionization of air is large enough that cesium concentration of less than one percent will not contribute significantly to the electron density.

Seeding with additives, which have a high electron affinity and hence form negative ions by electron capture [see for instance Massey, 1950; Branscomb, 1957; Bates, 1962] can be expected to decrease the electron concentration. The reaction for a given species $A$ is of the form:

$$
A+e^{-} \rightarrow A^{-}
$$

although other intermediate processes may be involved in some cases. The effect on the plasma is to replace a highly mobile electron by a less mobile heavy negative ion thereby markedly altering the electromagnetic properties of the plasma so that an $e-m$ wave interacts primarily with the remaining electrons in the plasma.

This seeding technique depends upon the selection of a molecule with a large electron capture cross section at the appropriate thermal conditions. For 
most elements, direct theoretical calculations are either too difficult or too tedious to permit quantitative evaluation of electron affinities. Hence it is difficult to rule out many species as potentially more appropriate than others. Table 1 shows a list of common electronegative seeding materials and some of their characteristic properties.

TABLE 1.-Characteristics of common electronegative gases

\begin{tabular}{|c|c|c|c|}
\hline $\begin{array}{l}\text { Electronegative } \\
\text { gas }\end{array}$ & $\begin{array}{l}\text { Attachment cross } \\
\text { section }\end{array}$ & $\begin{array}{c}\text { Electron energy } \\
\text { for max. } \\
\text { attachment }\end{array}$ & Reference \\
\hline $\mathrm{O}_{2} \ldots$ & $\begin{array}{l}\quad \stackrel{c m}{c} 2^{2} \\
\text { 1. } 3 \times 10^{-18} \\
\text { 2. } 25 \times 10^{-18}\end{array}$ & $\begin{array}{r}e v \\
6.2 \\
6.7\end{array}$ & $\begin{array}{l}\text { Buchelnikova [1959] } \\
\text { Craggs, Thorburn, } \\
\text { Tozer [1957] }\end{array}$ \\
\hline $\mathrm{CO}$ (forming $0^{-}$). & 2. $27 \times 10^{-18}$ & 10. 1 & Craggs \& Tozer [1958 \\
\hline $\mathrm{CO}_{2}$ (forming $0^{-}$). & $5.1 \times 10^{-19}$ & 7.8 & Craggs \& Tozer [1960] \\
\hline $\begin{array}{l}\mathrm{H}_{2} \mathrm{O} \\
\mathrm{HCl} \\
\mathrm{HBr}\end{array}$ & $\begin{array}{l}4.8 \times 10^{-18} \\
3.9 \times 10^{-18} \\
5.8 \times 10^{-17}\end{array}$ & $\begin{array}{r}6.4 \\
0.6 \\
.5\end{array}$ & Buchelnikova [1959] \\
\hline$I_{2 \ldots \ldots}$ & $3 \times 10^{-15}$ & $\sim 0$ & Biondi \& Fox [1958] \\
\hline $\mathrm{SF}_{6--}$ & $\begin{array}{r}\text { 1. } 2 \times 10^{-15} \\
5.7 \times 10^{-16} \\
1.2 \times 10^{-15} \\
\sim 10^{-15}\end{array}$ & $\begin{array}{l}\sim 0 \\
.01 \\
.01 \\
\sim 0\end{array}$ & $\begin{array}{l}\text { Craggs \& Meek [1962] } \\
\text { Buchelnikova [1959] } \\
\text { Buchelnikova [1958] } \\
\text { Hickam \& Fox [1956] }\end{array}$ \\
\hline $\mathrm{CCl}_{4} \ldots$ & $\begin{array}{l}\text { 1. } 3 \times 10^{-16} \\
1.7 \times 10^{-17}\end{array}$ & $\begin{array}{l}.02 \\
.02\end{array}$ & $\begin{array}{l}\text { Buchelnikova [1959] } \\
\text { Buchelnikova [1958[ }\end{array}$ \\
\hline
\end{tabular}

Very encouraging experimental results on seeding an argon plasma with sulfur hexafluoride $\left(\mathrm{SF}_{6}\right)$ as the electronegative gas have recently been obtained [Cloutier and Carswell, 1963; Carswell and Cloutier, 1964]. An indication of the striking change in the plasma created by the introduction of $\mathrm{SF}_{6}$ is shown in figure 9 where the microwave backscatter signal (at $9.2 \mathrm{Gc} / \mathrm{s}$ ) is plotted as function of the seeding ratio. For seeding ratios of the order $4 \times 10^{-4}$ the backscattered signal is reduced by at least a factor of 100 from the unseeded plasma. 'The capture process appears to be very efficient since this corresponds to approximately an equal number of electronegative modecu'es being iniected into the plasma as there are electrons in the plasma originally. Measurements performed using argon, nitrogen, and oxygen as the seed gases in argon were also studied and showed no effect on the plasma properties even at seeding rates which were a factor 10 greater than those used for the sulfur hexafluoride. Further experimental measurements under a variety of thermal conditions are still necessary before the technique can be established as suitable for appreciably modifying a reentry plasma and before realistic estimates of the required seed material can be made.

A second method of decreasing the electron concentration in a plasma is to alter the electron recombination time. Some measurements on the effect of introducing water vapor into a plasma have been reported by Kuhns [1962]. The influence of the water vapor was to decrease the electron density and hence reduce the attenuation of an rf signal propagating through the plasma. Considerably more investigation is required before the feasibility of this technique is established.

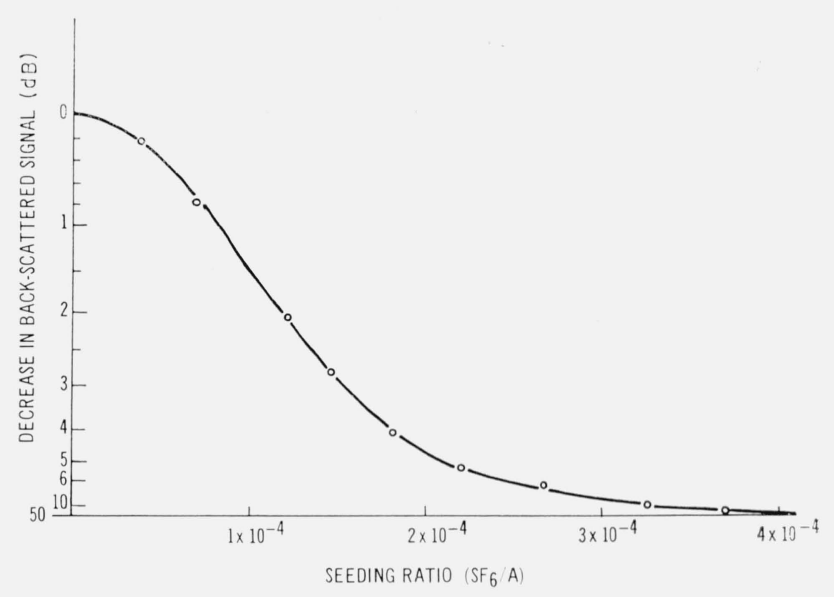

FiguRE 9. Variation of $9.2 \mathrm{Gc} / \mathrm{s}$ ratio signal backscattered from a plasma column for various seeding ratios (After Carswell and Cloutier [1964]).

A further physical process which may occur during the process of seeding is that the collision cross sections of the chemical additives will be greater than that of the initial species constituting the plasma. The net result is that the collision frequency of the plasma ensemble will be increased (since the collision frequencies of the various species may be considered as additive). This can result in a decrease in the reflecting properties of the plasma. However, more of the rf energy is absorbed in the plasma thus increasing the noise emission from the plasma and the net energy which is transmitted through the plasma is less than for a smaller collision frequency. In order to use this process for propagation of radio signals through a plasma it is necessary to decrease the collision frequency of the plasma (except at low radio frequencies and high collision frequencies as discussed earlier).

Removal of the concentration of free-electrons in a plasma by chemical injection of small (micron size) inert refractory material has also been suggested [Rosen, 1962]. The interaction of $e-m$ waves with such a medium is yet to be determined.

\subsection{Modification of Plasma by Aerodynamic Effects}

A major consideration is the location of the receiving or transmitting antenna on the vehicle. If possible this should be at regions of "low temperature" on the space craft and hence regions about which the smallest plasma density is created. Considerable control over this factor can be exercised by the design shape of the vehicle. A body with a sharp nose, for example, will create a weaker shock wave and hence generate a weaker plasma to engulf the vehicle. It may also be possible to protrude antennas outside the main plasma sheath such that the plasma formed on the antenna itself will be relatively thin. 
Any aerodynamic phenomena which tends to reduce the temperature on the body of a space vehicle and hence of the plasma near it will potentially reduce the ionization and thus the electron density of the plasma. Various cooling schemes such as forcing a fluid through a porous skin of the vehicle which is in contact with the hot plasma (transpiration cooling) have been suggested. The question is whether such cooling would be sufficient to reduce the electron density to a point where the plasma would have little effect on electromagnetic waves and hence communications, still remains to be answered.

\section{Conclusions}

A number of techniques - very high radio-frequency waves, low radio-frequency waves, imposition of static magnetic fields, chemical seeding and aerodynamic shaping show promise, under specific conditions, of permitting radio signals to penetrate through the plasma sheath generated by a hypersonic reentry vehicle. None of the methods are without major limitations. In most cases considerable experimental information is lacking in order to establish the technique on a firm practical basis.

\section{References}

Bachynski, M. P., T. W. Johnston, and I. P. Shkarofsky (1960), Electromagnetic properties of high temperature air, Proc. IRE 48, 347-356.

Bachynski, M. P., and B. W. Gibbs (1964), Electromagnetic wave propagation in anisotropic plasmas along the direction of a static magnetic field, RCA Victor Res. Rept. 7-801-833.

Bates, D. R. (1962), Atomic and molecular processes (Academic Press, New York, N.Y.).

Biondi, M. A., and R. E. Fox (1958), Dissociative attachment of electrons in iodine, Phys. Rev. 109, No. 6, 2012-2014.

Branscomb, L. M. (1957), Negative ions, Advances in Electronies and Electron Physics 9, 43-94.

Buchelnikova, N. S. (1958), Adhesion of slow electrons to $\mathrm{SF}_{6}$ and $\mathrm{CCl}_{4}$ molecules, Soviet Physics JETP $7,358-359$.

Buchelnikova, N. S. (1959), Cross-sections for the capture of slow electrons by $\mathrm{O}_{2}$ and $\mathrm{H}_{2} \mathrm{O}$ molecules and molecules of halogen components, Soviet Physics JETP 35, 783-791.

Carswell, A. I., and G. G. Cloutier (1964), Supersonic plasma streams seeded with electronegative gases, Phys. Fluids $\boldsymbol{\gamma}$, No. 4, 602-608.

Cloutier, G. G., and A. I. Carswell (1963), Plasma quenching by electronegative gas seeding, Phys. Rev. Letters 10, No. $8,327-329$.
Craggs, J. D., R. Thorburn, and B. A. Tozer (1957), The attachment of slow electrons in oxygen Proc. Roy. Soc. A-240, 473-483.

Craggs, J. D., and B. A. Tozer (1958), The attachment of slow electrons in carbon monoxide, Proc. Roy. Soc. A247, $337-345$.

Craggs, J. D., and B. A. Tozer (1960), The attachment of slow electrons in carbon dioxide, Proc. Roy. Soc. A254, $229-241$.

Craggs, J. D., and J. M. Meek (1962), Electrical discharges in sulfur hexafluoride, Gas Discharges and the Electrical Supply Industry, J. S. Forrest, P. R. Howard, D. J. Little, eds., pp. 338-347 (Butterworths, London).

Dellis, A. N., and J. M. Weaver (1964), Whistler mode propagation in a laboratory plasma, Proc. Phys. Soc. 83, $473-489$.

Evans, A., M. P. Bachynski, and A. G. Wacker (1962), Absorption in planetary atmospheres and sources of noise, ASD-TR-61-589, IV.

Fischer, S. T. (1963), Magneto-plasmas and re-entry radio blackout, Proc. IEEE 51, 1029-1030.

Flesher, G. T. (1960), On the elimination of communications dropout during space vehicle re-entry, NAECON Proc., Dayton, Ohio.

Hickam, M. A., and R. E. Fox (1956), Electron attachment in sulfur hexafluoride using monoenergetic electrons, J. Chem. Phys. 25, No. 4, 642-647.

Hochstim, A. R. (1961), Electron Concentration in closed form for high temperature air and air with additives, 'Electromagnetic Effects of Reentry,' pp. 79-93 (Pergamon Press, London).

Hodara, H., H. R. Raemer, and G. I. Cohn, (1960), A new approach to space communications, Proc. IXth Int. Astronautical Conf., Stockholm.

Hodara, H. (1961), The use of magnetic fields in the elimination of the reentry radio blackout, Proc. IRE 49, 1825-1830.

Kuhns, P. W. (1962), Microwave measurements of steadystate and decaying plasmas, IRE Trans. Space Electronics Telemetry, SET-8, No. 2, 173-178.

Mahaffey, D. W. (1963), microwave propagation through a plasma in a magnetic field, Phys. Rev. 129, No. 4, 14811488.

Massey, H. S. W. (1950), Negative Ions, 2d edition (Cambridge Univ. Press).

Mullin, C. R., and H. Hodara (1962), Elimination of reentry radio blackout, Proc. IRE 50, No. 10, 2108-2110.

Rosen, G. (1962), Method of removal of free electrons in a plasma, Phys. Fluids 5, 737.

Shkarofsky, I. P., and E. Brannen (1962), Generation and high level amplification of millimetre waves, ASD-TR$61-589$, II.

Warren, F. G. R., H. J. Moody, and T. V. Evangelatos (1962), System Considerations in Aerospace Communications, ASD-TR-61-589, VI. 